ESAIM: PROCEEDINGS AND SURVEYS, September 2018, Vol. 62, p. 17-29

Muhammad DAUHOO, Laurent DUMAS, Pierre GABRIEL and Pauline LAFITTE

\title{
NUMERICAL MODELING OF THE QUORUM SENSING IN A BACTERIAL BIOFILM
}

\author{
A. Blouza ${ }^{1}$ AND L. El Alaoui ${ }^{2}$
}

\begin{abstract}
In the present paper we propose a bi-dimensional non-linear reaction-diffusion model describing the action of antibiotics as well as quorum sensing inhibitors agents on the virulence of bacteria biofilms. We thus approximate the system by a standard finite element scheme in space and an implicit Euler method in time. We prove the existence and uniqueness of a solution to this system and prove its convergence to a weak solution of the model we introduce. We present numerical results for simulating the quorum sensing of the very human pathogenic bacteria Pseudomenas aeruginosa.

Résumé. Nous proposons dans le présent travail à un modèle bi-dimensionnel non linéaire de type réaction-diffusion décrivant l'action du traitement antibiotique conventionnel couplé à des agents inhibiteurs du système de communication intercellulaire, système qui contrôle la virulence de biofilms bactériens. Nous proposons une approximation en espace par éléments finis standard et en temps par un schéma d'Euler implicite. Nous montrons l'existence et l'unicité de cette approximation et montrons qu'elle converge vers une solution faible du modèle proposé. Nous illustrons notre approche par l'étude de la bactérie Pseudomenas aeruginosa connue pour être très pathogène chez l'homme.
\end{abstract}

\section{INTRODUCTION}

A biofilm is a structured community of microorganisms such as bacteria, algae and protozoa that live adherent to a surface or interface and which are surrounded in a self-produced extracellular polymers matrix.

Beneficial biofilms exist. Biobarriers to protect soil from contamination, bioremediation of waste sites or water-cleaning systems are examples of positive biofilms. But a lot of them have detrimental impacts on industrial environments (biofouling and biocorrosion) and particularly on public health. The cystic fibrosis and protheses infections and wide variety of nosocomial infections are caused by Pseudomonas aeruginosa or Staphylococcus aureus which are very human pathogenic bacteria.

In medical setting, growth and thus virulence of bacteria depend on nutrients and intercellular communication using biochemical signaling molecules. This cell-to-cell signaling mechanism is known as Quorum Sensing (QS) and provides a way of ensuring that certain genes mutate only when certain signal concentration has been reached.

We focus in the present work on the Pseudomonas aeruginosa complex QS system where the signal molecules are N-Acylated Homoserine Lactones (AHLs; e.g., N-(3-oxodo- decanoyl)-homoserine lactone denoted by 3-oxoC12-HSL) which bind to transcriptional regulator proteins (LasR) to activate the expression of target genes (See [1] for details).

\footnotetext{
${ }^{1}$ LMRS, CNRS-Université de Rouen Normandie, 76801 Saint-Etienne-du-Rouvray, France, email: adel.blouza@univ-rouen.fr

2 Université Paris 13, Sorbonne Paris Cité, LAGA, CNRS (UMR 7539), Laboratoire d'excellence Inflamex, F-93430, Villetaneuse, France. email: elalaoui@math.univ-paris13.fr
}

(C) EDP Sciences, SMAI 2018 
As described in [1], QS behavior in an infection setting is as follows: in the early stages of infection, at low cell density, the pathogen remains relatively unnoticed by the immune system, but as numbers increase the bacteria can use their density sensing mechanism to switch on virulence genes rapidly and in unison, resulting in the production of a combination of virulence factors which may overwhelm host defenses. QS works by diffusion of one or more self-generated signal molecules, often produced auto-inductively. The chemical class of the signal molecule depends on the bacterial species.

Bacterial species such as Pseudomonas aeruginosa or Staphylococcus aureus increase their resistance to antibiotics. For blocking this intercellular communication, Williams in [10] had already the idea to target the quorum sensing for antibacterial chemotherapy. In [1], the authors have modeled the effect of an AHL-degrading enzyme as well as the effect of LasR-degrading drug on the QS together with antibiotic treatment to attenuate the virulence of those pathogenic bacteria.

In the present study, we consider a system of PDEs, in bi-dimensional setting, for the action on QS of Pseudomonas aeruginosa combined with an antibiotic and taking the reaction kinetics of bacterial cells to be those of ([1], [2]) and the diffusion terms as introduced in [7]. However, in contrast to these authors, we allow the bacterial cells to grow according to Monod's law and take into account the diffusion of LasR and antibiotic as well. Several papers deal with the mathematical modelling of the quorum sensing, one cite for instance $[4-7,10]$.

In section 2 of this paper, we introduce our reaction-diffusion equations modeling the effect antibiotic together with LasR-degrading drug on the QS system of $P$. aeruginosa.

In section 3, We write the corresponding discret weak formulation of equations introduced in the previous section using implicit Euler scheme in time and finite element method in space and prove its well-posedness. We also provide a qualitative analysis to study the effect of agent capable to reduce the LasR signal transduction protein of the QS system of Pseudomonas aeruginosa. A simplified schematic of the gene network involved in the primary QS system is given in Fig.1 below.

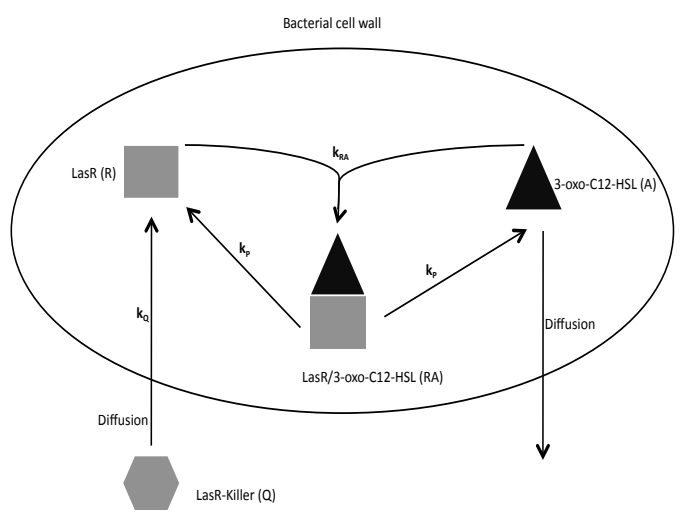

FiguRE 1. Schematic representation of the network involved in the QS system of Pseudomonas aeruginosa.

\section{The MATHEMATiCAL MODEL}

In this section we propose a bi-dimensional mathematical modeling of the quorum sensing in a bacterial biofilm. The model is derived from mass balance principles that describe the nutrient concentration, biomass concentration, LasR and AHL concentrations as well as the QS inhibitor and the antibiotic concentrations within the biofilm.

The concentration variables of the model are listed in the Table 1 below, most of the values are from $[1,4,5]$ and some are assumed. The model we introduce consists in a coupled system of nonlinear diffusion-reaction equations in $\Omega \times(0, T), T>0$ where $\Omega \subset \mathbb{R}^{2}$ is a bounded domain and $\partial \Omega=\Gamma_{N} \cup \Gamma_{D}$, with $\Gamma_{N} \cap \Gamma_{D}=\emptyset$, 
denotes its boundary.

\begin{tabular}{l|c} 
Concentration & Variable \\
\hline down-regulated cell & $X$ \\
up-regulated cell & $Y$ \\
LasR & $R$ \\
3-oxo-C12-HSL cell & A \\
Antibiotic & $V$ \\
Nutrient & $S$ \\
LasR-degrading drug & $Q$
\end{tabular}

TABLE 1. Notation used for concentration variables.

All the parameters involved in the following equations are defined in Table 2 and are positive. We introduce the Monod's function that is: $\tilde{g}(u, v)=\mu \frac{u}{k+u} v=\mu g(u, v)$, where $k$ is the half-saturation constant. It is the value for which $u=k$ implies a $v$-growth at half the maximum growth rate $\mu$. Monod kinetics used here implies a saturation effect. In other words, even if the substrate $u$ is available in abundance the specified maximum growth cannot be exceeded.

- The substrate $S$ is injected in $\Omega$ through a part of its boundary $\Gamma_{D}$. The nutrient allows to diffuse into and out the bacteria cells at the same rate and is consumed by the total biomass $X+Y$ owing to the Monod's kinetics. Hence, the evolution of the nutrient is described as:

$$
\partial_{t} S=d_{S} \Delta S-k_{1} \frac{S}{k_{2}+S}(X+Y)
$$

- From the mass balance principle, the evolution of the total biomass $M$ is modeled by

$$
\partial_{t} M=\nabla(D(M) \nabla M)+G(S, M)
$$

where $G(S, M)$ expresses the growth of the biomass and $D(M) \approx P^{\prime}(M)$ with $P$ the unknown biofilm pressure. In this work we assume that $D(M)$ does not depend on $M$. This assumption is not too restrictive since we are not concerned by the biofilm shape.

- The biomass (down-regulated and up-regulated cells) is produced by the consumption of nutrients according to the Monod's law. Cells die naturally, the down-regulated cells are killed by the antibiotic, and the downregulated cells are converted to up-regulated cells due to the increasing of the AHL concentration. The up-regulated cells are converted to down-regulated cells at a constant rate. Hence, it holds that

$$
\begin{aligned}
& \partial_{t} X=d \Delta X+k_{3} \frac{S}{k_{2}+S} X-k_{5} A^{m} X+k_{5} Y-k_{4} X-k_{V} X V \\
& \partial_{t} Y=d \Delta Y+k_{3} \frac{S}{k_{2}+S} Y+k_{5} A^{m} X-k_{5} Y-k_{4} Y
\end{aligned}
$$

Thus, the biofilm is defined by the domain $\{x \in \Omega ; X(x, t)+Y(x, t)>0\}$.

- The AHL molecules diffuse through the domain, the AHL molecules decay abiotically at rate $\gamma$. Downregulated cells produce the signaling molecule at rate $\alpha$, whereas up-regulated cells produce it at the increased rate $\alpha+\beta$, where is one order of magnitude larger than $\alpha$. The signaling molecule production rate of the 


\begin{tabular}{c|c|c} 
Symbol & Parameter & Value \\
\hline$k_{1}$ & maximum specific consumption rate & 794 \\
$k_{2}$ & Monod half saturation & 0.2 \\
$k_{3}$ & maximum specific growth rate & 1 \\
$k_{4}$ & lysis rate & 0.0667 \\
$k_{5}$ & quorum sensing regulation rate & 52.7 \\
$k_{R}$ & LasR degradation rate & 0.1 \\
$k_{L}$ & AHL degradation rate & 0.01 \\
$k_{V}$ & Antibiotic reaction rate & 0.1 \\
$k_{Q}$ & QSB reaction rate & 0.05 \\
$l_{V}$ & antibiotic degradation rate & 0.1 \\
$d$ & biomass motility & $1 \mathrm{e}-7$ \\
$d_{S}$ & nutrient diffusion & 1.67 \\
$d_{R}$ & LasR diffusion & 0.1 \\
$d_{V}$ & antibiotic diffusion & 0.025 \\
$d_{Q}$ & QSB diffusion & 1 \\
$d_{2}$ & Diffusion coefficient of autoinductor & 12.9 \\
$\alpha$ & AHL production rate of down-regulated cells & 92 \\
$\beta$ & Increased AHL production activity by up-regulated cells & 920 \\
$\gamma$ & Abiotic AHL decay rate & 0.022 \\
$m$ & polymerisation degree & 2.5
\end{tabular}

TABLE 2. Dimensionless model parameters used in the system (1)-(8), adapted from [6].

up-regulated cells is higher than the abiotic decay rate, i.e., $\alpha+\beta>\gamma$. See Table 2 .

$$
\partial_{t} A=d_{2} \Delta A-\gamma A+\alpha \frac{R A}{k_{L}+R A} X+(\alpha+\beta) \frac{R A}{k_{L}+R A} Y .
$$

- The LasR protein is involved in the production of the LasR/3-oxo-C12-HSL (whose concentration is proportional to RA, [1]), degrades naturally at a constant rate $k_{R}$, and is blocked by the QSB agent. Hence, we get

$$
\partial_{t} R=d_{R} \Delta R-k_{R} R+k_{3} \frac{R A}{k_{R}+R A}-k_{3} \frac{X+Y}{k_{2}+X+Y} R+k_{Q} R Q .
$$

- The antibiotic diffuses in the culture at rate $d_{V}$, degrades with rate $l_{V}$, and reacts with the down-regulated cell at rate $k_{V}$. Thus, we obtain

$$
\partial_{t} V=d_{V} \Delta V-l_{V} V-k_{V} X V
$$

- Finally, we assume that the QSB agent diffuses in the culture at rate $d_{Q}$, degrades with rate $l_{Q}$, and reacts with the down-regulated cell at rate $k_{Q}$.

$$
\partial_{t} Q=d_{Q} \Delta Q-l_{Q} Q-k_{Q} R Q
$$


We complete the system by the boundary and initial conditions below.

$$
\begin{aligned}
& \nabla X(x, t) \cdot \nu=\nabla Y(x, t) \cdot \nu=\nabla R(x, t) \cdot \nu=\nabla A(x, t) \cdot \nu=0, \quad(x, t) \in \partial \Omega \times(0, T), \\
& \nabla S(x, t) \cdot \nu=\nabla V(x, t) \cdot \nu=\nabla Q(x, t) \cdot \nu=0, \quad(x, t) \in \Gamma_{N} \times(0, T), \\
& S(x, t)=S_{D}, V(x, t)=V_{D}, Q(x, t)=Q_{D}, \quad(x, t) \in \Gamma_{D} \times(0, T), \\
& X(x, 0)=X_{0}, Y(x, 0)=0, R(x, 0)=R_{0}, A(x, 0)=A_{0} \quad x \in \Omega, \\
& S(x, 0)=V(x, 0)=Q(x, 0)=0, \quad x \in \Omega .
\end{aligned}
$$

with $X_{0}, R_{0}, A_{0}, S_{0}, V_{0}, Q_{0}$ are positive constants.

\section{Numerical APPROXIMATION}

In this section we propose a numerical approximation of the nonlinear system of partial differential equations described in the previous section. We discretize the system in space by finite elements and in time by an implicit Euler scheme.

For a sake of simplicity we present the discretization of the nonlinear system describing the tempo-spatial evolution of the biomass, the AHL, the LasR and the substrate concentrations.

Le $0=t_{0}<t_{1}<\cdots<t_{N-1}<t_{N}=T$ be a partitioning of $[0, T]$ into variable time steps $\tau_{n}=t_{n}-t_{n-1}, n=$ $1, \ldots, N$. We set $\tau=\max _{1 \leq n \leq N} \tau_{n}$. We introduce $\left(\mathcal{T}_{h}\right)_{h}$ a shape-regular family of triangulations of $\Omega$. For a triangle $\kappa \in \mathcal{T}_{h}$, let $h_{\kappa}$ be its diameter and set $h=\max _{\kappa \in \mathcal{T}_{h}} h_{\kappa}$. For any subdomain $\omega$ of $\Omega$ we define by $\mathbb{P}^{1}(\omega)$ the space of polynomials on $\omega$ with degree $\leq 1$. We denote by $(\cdot, \cdot)_{\omega}$ the usual $L^{2}$ product on $\omega$ and by $\|\cdot\|_{\omega}$ the associated norm. Furthermore, we denote by $|\cdot|_{1, \omega}$ the semi-norm on $H^{1}(\omega)$. For ease of notation, when $\omega=\Omega$ we drop the subscript.

We introduce the $P^{1}$-finite element spaces

$$
\mathbb{P}^{1}\left(\mathcal{T}_{h}\right)=\left\{v_{h} \in \mathcal{C}^{0}(\Omega) ; \forall \kappa \in \mathcal{T}_{h},\left.v_{h}\right|_{\kappa} \in P^{1}(\kappa)\right\}, \quad \mathbb{P}_{\beta}^{1}\left(\mathcal{T}_{h}\right)=\left\{v_{h} \in \mathbb{P}^{1}\left(\mathcal{T}_{h}\right),\left.v_{h}\right|_{\Gamma_{D}}=\beta\right\}
$$

and we set

$$
\mathcal{W}_{h}=\left\{v_{h} \in \mathbb{P}^{1}\left(\mathcal{T}_{h}\right) ; v_{h} \geq 0\right\}, \quad \text { and } \quad \mathcal{W}_{h}^{\beta}=\left\{v_{h} \in \mathbb{P}_{\beta}^{1}\left(\mathcal{T}_{h}\right) ; v_{h} \geq 0\right\}
$$

We consider the problem $\left(\mathcal{P}_{\mathbf{h}}\right)$ : For $\left(S_{h}^{n}, A_{h}^{n}, X_{h}^{n}, Y_{h}^{n}, R_{h}^{n}\right)$ given in $\mathbb{P}^{1}\left(\mathcal{T}_{h}\right) \times \mathbb{P}_{S_{D}}^{1}\left(\mathcal{T}_{h}\right) \times \mathbb{P}^{1}\left(\mathcal{T}_{h}\right) \times \mathbb{P}^{1}\left(\mathcal{T}_{h}\right) \times \mathbb{P}^{1}\left(\mathcal{T}_{h}\right)$, we seek

such that

$$
\left(S_{h}^{n+1}, A_{h}^{n+1}, X_{h}^{n+1}, Y_{h}^{n+1}, R_{h}^{n+1}\right) \in \mathbb{P}_{S_{D}}^{1}\left(\mathcal{T}_{h}\right) \times \mathbb{P}^{1}\left(\mathcal{T}_{h}\right) \times \mathbb{P}^{1}\left(\mathcal{T}_{h}\right) \times \mathbb{P}^{1}\left(\mathcal{T}_{h}\right) \times \mathbb{P}^{1}\left(\mathcal{T}_{h}\right)
$$

$$
\begin{aligned}
& \int_{\Omega} \frac{\left(S_{h}^{n+1}-S_{h}^{n}\right)}{\tau_{n}} v_{h}^{S} d x+\int_{\Omega}\left[d_{S} \nabla S_{h}^{n+1} \cdot \nabla v_{h}^{S}+k_{1} g\left(S_{h}^{n+1}, X_{h}^{n}+Y_{h}^{n}\right) v_{h}^{S}\right] d x=0 \\
& \int_{\Omega} \frac{\left(A_{h}^{n+1}-A_{h}^{n}\right)}{\tau_{n}} v_{h}^{A} d x+\int_{\Omega}\left[d_{2} \nabla A_{h}^{n+1} \cdot \nabla v_{h}^{A}+\gamma A_{h}^{n+1} v_{h}^{A}\right] d x=\int_{\Omega} \alpha \frac{R_{h}^{n} A_{h}^{n+1}}{K_{L}+R_{h}^{n} A_{h}^{n+1}} X_{h}^{n} v_{h}^{A} d x+\int_{\Omega}(\alpha+\beta) \frac{R_{h}^{n} A_{h}^{n+1}}{K_{L}+R_{h}^{n} A_{h}^{n+1}} Y_{h}^{n} v_{h}^{A} d x \\
& \int_{\Omega} \frac{\left(X_{h}^{n+1}-X_{h}^{n}\right)}{\tau_{n}} v_{h}^{X}+\int_{\Omega}\left[d \nabla X_{h}^{n+1} \cdot \nabla v_{h}^{X}+\left(k_{4} X_{h}^{n+1}+k_{5}\left|A_{h}^{n+1}\right|^{m} X_{h}^{n+1}\right) v_{h}^{X}\right] d x=\int_{\Omega}\left(k_{3} g\left(S_{h}^{n+1}, X_{h}^{n}\right)+k_{5} Y_{h}^{n}\right) v_{h}^{X} d x \\
& \int_{\Omega} \frac{\left(Y_{h}^{n+1}-Y_{h}^{n}\right)}{\tau_{n}} v_{h}^{Y} d x+\int_{\Omega}\left[d \nabla Y_{h}^{n+1} \cdot \nabla v_{h}^{Y}+\left(k_{4}+k_{5}\right) Y_{h}^{n+1} v_{h}^{Y}\right] d x=\int_{\Omega}\left(k_{3} g\left(S_{h}^{n+1}, Y_{h}^{n+1}\right)+k_{5}\left|A_{h}^{n+1}\right|^{m} X_{h}^{n+1}\right) v_{h}^{Y} d x
\end{aligned}
$$

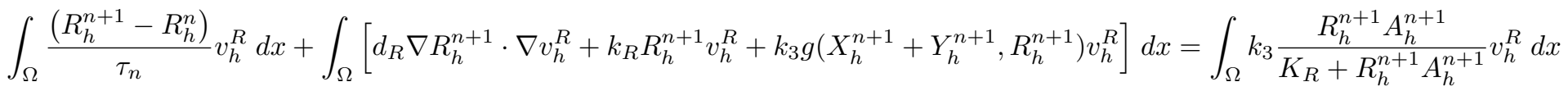


for all $\left(v_{h}^{S}, v_{h}^{A}, v_{h}^{X}, v_{h}^{Y}, v_{h}^{R}\right) \in \mathbb{P}_{0}^{1}\left(\mathcal{T}_{h}\right) \times \mathbb{P}^{1}\left(\mathcal{T}_{h}\right) \times \mathbb{P}^{1}\left(\mathcal{T}_{h}\right) \times \mathbb{P}^{1}\left(\mathcal{T}_{h}\right) \times \mathbb{P}^{1}\left(\mathcal{T}_{h}\right)$.

Remark.- Let us note that the numerical scheme $\left(\mathcal{P}_{\mathbf{h}}\right)$ allows to solve successively nonlinear equations instead of solving a fully nonlinear system.

Theorem 3.1. Let $\left(S_{h}^{n}, A_{h}^{n}, X_{h}^{n}, Y_{h}^{n}, R_{h}^{n}\right)$ given in $\mathcal{W}_{h}^{S_{D}} \times \mathcal{W}_{h} \times \mathcal{W}_{h} \times \mathcal{W}_{h} \times \mathcal{W}_{h}$. Then for all $h, \tau_{n}>0$, there exists a unique solution $\left(S_{h}^{n+1}, A_{h}^{n+1}, X_{h}^{n+1}, Y_{h}^{n+1}, R_{h}^{n+1}\right) \in \mathcal{W}_{h}^{S_{D}} \times \mathcal{W}_{h} \times \mathcal{W}_{h} \times \mathcal{W}_{h} \times \mathcal{W}_{h}$ of $\left(\mathcal{P}_{\mathbf{h}}\right)$.

Proof. We denote by $\left\{\phi_{i}\right\}_{1 \leq i \leq N}$ the usual basis functions of $\mathbb{P}_{0}^{1}\left(\mathcal{T}_{h}\right)\left(\operatorname{dim}\left(\mathbb{P}_{0}^{1}\left(\mathcal{T}_{h}\right)=N\right)\right.$. For any $W=$ $\sum_{j=1}^{N} W_{j} \phi_{j} \in \mathbb{P}_{0}^{1}\left(\mathcal{T}_{h}\right)$ we define $\underline{W}=\left(W_{1}, \ldots, W_{N}\right)^{T} \in \mathbb{R}^{N}$. We then set $F_{S}^{n}: \mathbb{R}^{N} \times \mathbb{R}^{N} \rightarrow \mathbb{R}^{N}$ defined by :

$$
\left[F_{S}^{n}(\underline{W}, \underline{Z})\right]_{j}=\left(W, \phi_{j}\right)+\tau_{n} d\left(\nabla W, \nabla \phi_{j}\right)+\tau_{n}\left(f(W) Z, \phi_{j}\right), \quad j=1, \cdots N
$$

For $S^{n}$ and $X^{n}$ given in $\mathcal{W}_{h}^{S_{D}}$ and $\mathcal{W}_{h}^{0}$, respectively, we write the first equation of $\left(\mathcal{P}_{h}\right)$, where $d=d_{S}$, as:

$$
F_{S}^{n}\left(\underline{S}^{n+1}, \underline{X}^{n}+\underline{Y}^{n}\right)=\underline{G}_{S}^{n} \in \mathbf{R}_{\geq 0}^{N}
$$

with $\left[\underline{G}_{S}^{n}\right]_{j}:=\left(S^{n}, \phi_{j}\right)$. One can prove that, for every fixed $Z \in \mathbb{R}^{N}$, we have that $F_{S}^{n}(., Z): \mathbb{R}^{N} \rightarrow \mathbb{R}^{N}$ is an isotone homeomorphism. We refer to [3] for more details, and from $F_{S}^{n}\left(\underline{0}, \underline{X}^{n}\right)=\underline{0}$ we deduce the existence and uniqueness solution $S_{h}^{n}$ in $\mathcal{W}_{h}^{S_{D}}$ solving the first equation of $\left(\mathcal{P}_{h}\right)$.

In the same way we prove the existence and uniqueness of $\left(A_{h}^{n}, X_{h}^{n}, Y_{h}^{n}, R_{h}^{n}\right)$ in $\left[\mathcal{W}_{h}\right]^{4}$.

For any $Z_{h}^{n+1} \in \mathcal{W}$, we set

$$
\mathcal{F}\left(Z^{n+1}\right)=\sum_{n=1}^{N} \tau_{n}\left|\frac{Z^{n+1}-Z^{n}}{\tau_{n}}\right|^{2}+\max _{n}\left|Z^{n+1}\right|_{1}^{2}+\sum_{n=1}^{N} \tau_{n}\left|Z^{n+1}-Z^{n}\right|_{1}^{2} .
$$

Lemma 3.2. For all $h>0$ and for all time partitions $\left\{\tau_{n}\right\}_{n}$, the solution $\left(S_{h}^{n}, A_{h}^{n}, X_{h}^{n}, Y_{h}^{n}, R_{h}^{n}\right)$ of $\left(\mathcal{P}_{h}\right)$ satisfies

$$
\mathcal{F}\left(R_{h}^{n+1}\right)+\mathcal{F}\left(S_{h}^{n+1}\right)+\mathcal{F}\left(X_{h}^{n+1}\right)+\mathcal{F}\left(Y_{h}^{n+1}\right)+\mathcal{F}\left(A_{h}^{n+1}\right) \leq C\left(\left|R_{h}^{0}\right|_{1}^{2}+\left|S_{h}^{0}\right|_{1}^{2}+\left|X_{h}^{0}\right|_{1}^{2}+\left|Y_{h}^{0}\right|_{1}^{2}+\left|A_{h}^{0}\right|_{1}^{2}+T\right) .
$$

The above bound is obtained by using $Z_{h}^{n+1}-Z_{h}^{n}$ as test function in each equation of $\left(\mathcal{P}_{h}\right)$. Here $Z_{h}^{n}$ denotes arbitrarily $S_{h}^{n}, A_{h}^{n}, X_{h}^{n}, Y_{h}^{n}$, or $R^{n}$.

Let us now state that the solution of the numerical scheme converges to a weak solution of $(\mathcal{P})$.

We set $\Omega_{T}=\Omega \times(0, T)$ and we introduce the following notation

$$
Z(t)=\frac{t-t^{n}}{\tau_{n}} Z^{n+1}+\frac{t^{n+1}-t}{\tau_{n}} Z^{n}, \quad t \in\left[t^{n}, t^{n+1}\right], n \geq 1
$$

and

$$
Z^{+}(t)=Z^{n+1}, Z^{-}(t)=Z^{n}, \quad t \in\left[t^{n}, t^{n+1}\right], n \geq 1 .
$$


Using this notation the problem $\left(\mathcal{P}_{h}\right)$ reads as: Seek $\left(S_{h}, A_{h}, X_{h}, Y_{h}, R_{h}\right) \in\left[\mathcal{C}^{0}\left([0, T] \times \mathcal{W}_{h}\right)\right]^{5}$ such that

$$
\begin{aligned}
& \int_{\Omega_{T}} \frac{\partial S_{h}}{\partial t} v_{h}+\int_{\Omega_{T}} d_{S} \nabla S_{h} \cdot \nabla v_{h}=\int_{\Omega_{T}} k_{1} g\left(S_{h}, X_{h}+Y_{h}\right) v_{h} \\
& \int_{\Omega_{T}} \frac{\partial A_{h}}{\partial t} v_{h}+\int_{\Omega_{T}} d_{2} \nabla A_{h} \cdot \nabla v_{h}+\gamma \int_{\Omega_{T}} A_{h} v_{h}=\int_{\Omega_{T}} \alpha \frac{R_{h} A_{h}}{K_{L}+R_{h} A_{h}} X_{h} v_{h}+\int_{\Omega_{T}}(\alpha+\beta) \frac{R_{h} A_{h}}{K_{L}+R_{h} A_{h}} Y_{h} v_{h}, \\
& \int_{\Omega_{T}} \frac{\partial X_{h}}{\partial t} v_{h}+\int_{\Omega_{T}} d \nabla X_{h} \cdot \nabla v_{h}+\int_{\Omega_{T}}\left(k_{4} X_{h}+k_{5}\left|A_{h}\right|^{m} X_{h}\right) v_{h}=\int_{\Omega_{T}}\left(k_{3} g\left(S_{h}, X_{h}\right)+k_{5} Y_{h}\right) v_{h}, \\
& \int_{\Omega_{T}} \frac{\partial Y_{h}}{\partial t} v_{h}+\int_{\Omega_{T}} d \nabla Y_{h} \cdot \nabla v_{h}+\int_{\Omega_{T}}\left(k_{4}+k_{5}\right) Y_{h} v_{h}=\int_{\Omega_{T}}\left(k_{3} g\left(S_{h}, Y_{h}\right)+k_{5}\left|A_{h}\right|^{m} X_{h}\right) v_{h}, \\
& \int_{\Omega_{T}} \frac{\partial R_{h}}{\partial t} v_{h}+\int_{\Omega_{T}} d_{R} \nabla R_{h} \cdot \nabla v_{h}+\int_{\Omega_{T}} k_{R} R_{h} v_{h}+\int_{\Omega_{T}} k_{3} g\left(X_{h}+Y_{h}, R_{h}\right) v_{h}=\int_{\Omega_{T}} k_{3} \frac{R_{h} A_{h}}{K+R_{h} A_{h}} v_{h} .
\end{aligned}
$$

On introducing

$$
\mathcal{G}(Z)=\left\|Z^{ \pm}\right\|_{L^{\infty}\left(\Omega_{T}\right)}^{2}+\left\|Z^{ \pm}\right\|_{L^{\infty}\left(0, T ; H^{1}(\Omega)\right)}^{2}+\left\|\frac{\partial Z}{\partial t}\right\|_{L^{2}\left(\Omega_{T}\right)}^{2}+\tau^{-1}\left\|Z^{+}-Z^{-}\right\|_{L^{2}\left(0, T ; H^{1}(\Omega)\right)}^{2},
$$

where $Z^{ \pm}$is an abbreviation for "with" and "without" the superscripts "+" and " - ", the lemma (3.2) yields that

$$
\mathcal{G}\left(R_{h}\right)+\mathcal{G}\left(S_{h}\right)+\mathcal{G}\left(X_{h}\right)+\mathcal{G}\left(Y_{h}\right)+\mathcal{G}\left(A_{h}\right) \leq C(T)
$$

In the following $Z_{h}=S_{h}, A_{h}, X_{h}, Y_{h}$, or $R_{h}$ and $Z=S, A, X, Y$ or $R$.

From the inequality (13) we infer the convergence result stated in the lemma below:

Lemma 3.3. Let us assume that there is a constant $C>0$ such that $\tau h<C$. Then, there exists a subsequence $\{Z\}_{h}$ and a function $z \in L^{\infty}\left(\Omega_{T}\right) \cap H^{1}\left(0, T ; L^{2}(\Omega)\right)$ such that as $h \rightarrow 0$

$$
\begin{aligned}
& Z, Z^{ \pm} \longrightarrow z \quad \text { weak- } \star \text { in } L^{\infty}\left(\Omega_{T}\right) \\
& \frac{\partial Z}{\partial t} \longrightarrow \frac{\partial z}{\partial t} \quad \text { weakly } L^{2}\left(\Omega_{T}\right) \\
& Z, Z^{ \pm} \longrightarrow z \quad \text { weak- } \text { in } L^{\infty}\left(0, T ; H^{1}(\Omega)\right) \\
& Z, Z^{ \pm} \longrightarrow z \text { strongly in } L^{2}\left(\Omega_{T}\right) \text { and a.e. in } \Omega_{T} \text {. }
\end{aligned}
$$


Theorem 3.4. Under the assumption of Lemma 3.3, and $Z_{0} \in H^{2}(\Omega)$, the solution of $\left(\mathcal{P}_{h}\right)$ converges to the solution of the following weak form of (1)-(5).

Seek $(S, A, X, Y, R) \in L^{\infty}\left(0, T ; H^{2}(\Omega) \cap H_{S_{D}}^{1}(\Omega) \cap \mathcal{C}(\bar{\Omega}) \times\left[L^{\infty}\left(0, T ; H^{2}(\Omega) \cap \mathcal{C}(\bar{\Omega})\right]^{4}\right.\right.$ such that :

$$
\begin{aligned}
& \int_{\Omega_{T}} \frac{\partial S}{\partial t} v^{S}+\int_{\Omega_{T}} d_{S} \nabla S \cdot \nabla v^{S}=\int_{\Omega_{T}} k_{1} g(S, X+Y) v^{S} \\
& \int_{\Omega_{T}} \frac{\partial A}{\partial t} v^{A}+\int_{\Omega_{T}} d_{2} \nabla A \cdot \nabla v^{A}+\gamma \int_{\Omega_{T}} A v^{A}=\int_{\Omega_{T}} \alpha \frac{R A}{K_{L}+R A} X v^{A}+\int_{\Omega_{T}}(\alpha+\beta) \frac{R A}{K_{L}+R A} Y v^{A} \\
& \int_{\Omega_{T}} \frac{\partial X}{\partial t} v^{X}+\int_{\Omega_{T}} d \nabla X \cdot \nabla v^{X}+\int_{\Omega_{T}}\left(k_{4} X+k_{5}|A|^{m} X\right) v^{X}=\int_{\Omega_{T}}\left(k_{3} g(S, X)+k_{5} Y\right) v^{X} \\
& \int_{\Omega_{T}} \frac{\partial Y}{\partial t} v^{Y}+\int_{\Omega_{T}} d \nabla Y \cdot \nabla v^{Y}+\int_{\Omega_{T}}\left(k_{4}+k_{5}\right) Y v^{Y}=\int_{\Omega_{T}}\left(k_{3} g(S, Y)+k_{5}|A|^{m} X\right) v^{Y} \\
& \int_{\Omega_{T}} \frac{\partial R}{\partial t} v^{R}+\int_{\Omega_{T}} d_{R} \nabla R \cdot \nabla v^{R}+\int_{\Omega_{T}} k_{R} R v^{R}+\int_{\Omega_{T}} k_{3} g(X+Y, R) v^{R}=\int_{\Omega_{T}} k_{3} \frac{R A}{K+R A} v^{R} .
\end{aligned}
$$

for all $\left(v^{S}, v^{A}, v^{X}, v^{Y}, v^{R}\right) \in L^{2}\left(0, T ; H_{0}^{1}(\Omega) \times\left[L^{2}\left(0, T ; H^{1}(\Omega)\right]^{4}\right.\right.$.

\section{NumericAl RESUlts}

The computational domain we consider is $\Omega_{T}=[0,1] \times[0,0.5] \times[0,20]$. The boundary $\Gamma_{D}$ on which the substrate, the antibiotic and the QSB agent are applied corresponds here to the top boundary of the computational domain. At $t=0$ the biofilm domain is defined by $\Omega_{b}(0)=\left\{\left(x_{1}, x_{2}\right), \in \Omega ; x_{2} \geq 0, x_{1}^{2}+x_{2}^{2}=0.01\right\}$ and contained down-regulated biomass only.

In Figure 2 we show the spatial representation of down-regulated (column 1 and 3) and up-regulated cells (column 2 and 4 ) at time $t=10$ and $t=15$. At the first line we show the representation when no antibiotic nor QSB agent are applied, the second line corresponds to the case where only antibiotic is applied and third line corresponds to the case where antibiotic and QSB agent act. One observe how antibiotic and QSB agent allow to reduce the quantity of bacteria and also to modify the spatial expansion of bacteria. 

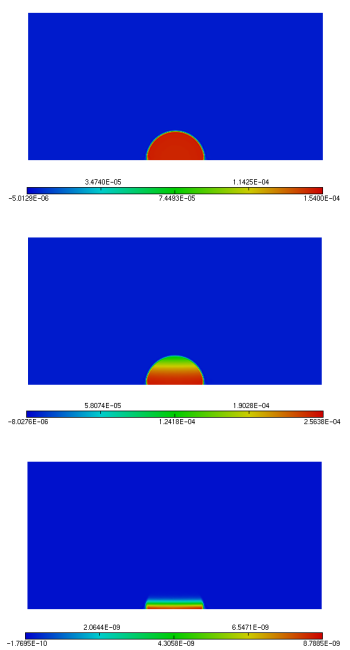
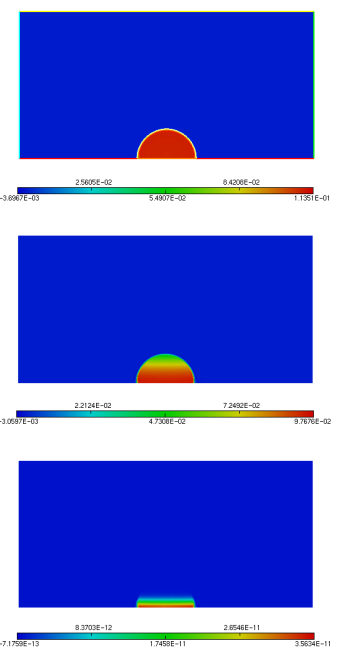
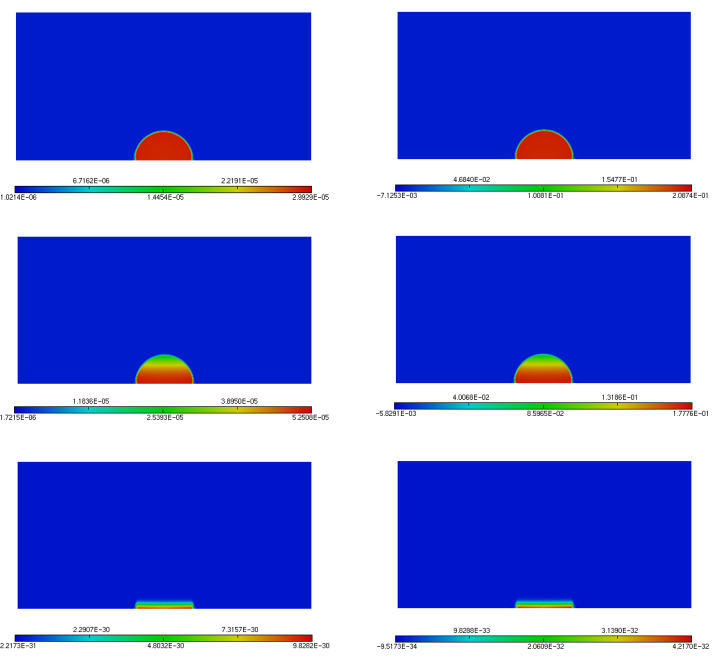

Figure 2. Spatial representation of down- and up-regulated cells at time $t=10$ and $t=15$.

The numerical results we present below concern the evolution of the total amount of each variable with respect to time. We set

$$
\begin{aligned}
X_{\text {Total }}(t) & =\frac{1}{|\Omega|} \int_{\Omega} X(x, t) d x, & Y_{\text {Total }}(t) & =\frac{1}{|\Omega|} \int_{\Omega} Y(x, t) d x, \\
A_{\text {Total }}(t) & =\frac{1}{|\Omega|} \int_{\Omega} A(x, t) d x, & R_{\text {Total }}(t) & =\frac{1}{|\Omega|} \int_{\Omega} R(x, t) d x .
\end{aligned}
$$

The quantity of antibiotic applied at the top of the domain is $V_{D}=10$ and the quantity of QSB agent applied at the top of the domain, $\Gamma_{D}$, is $Q_{D}=10$. 

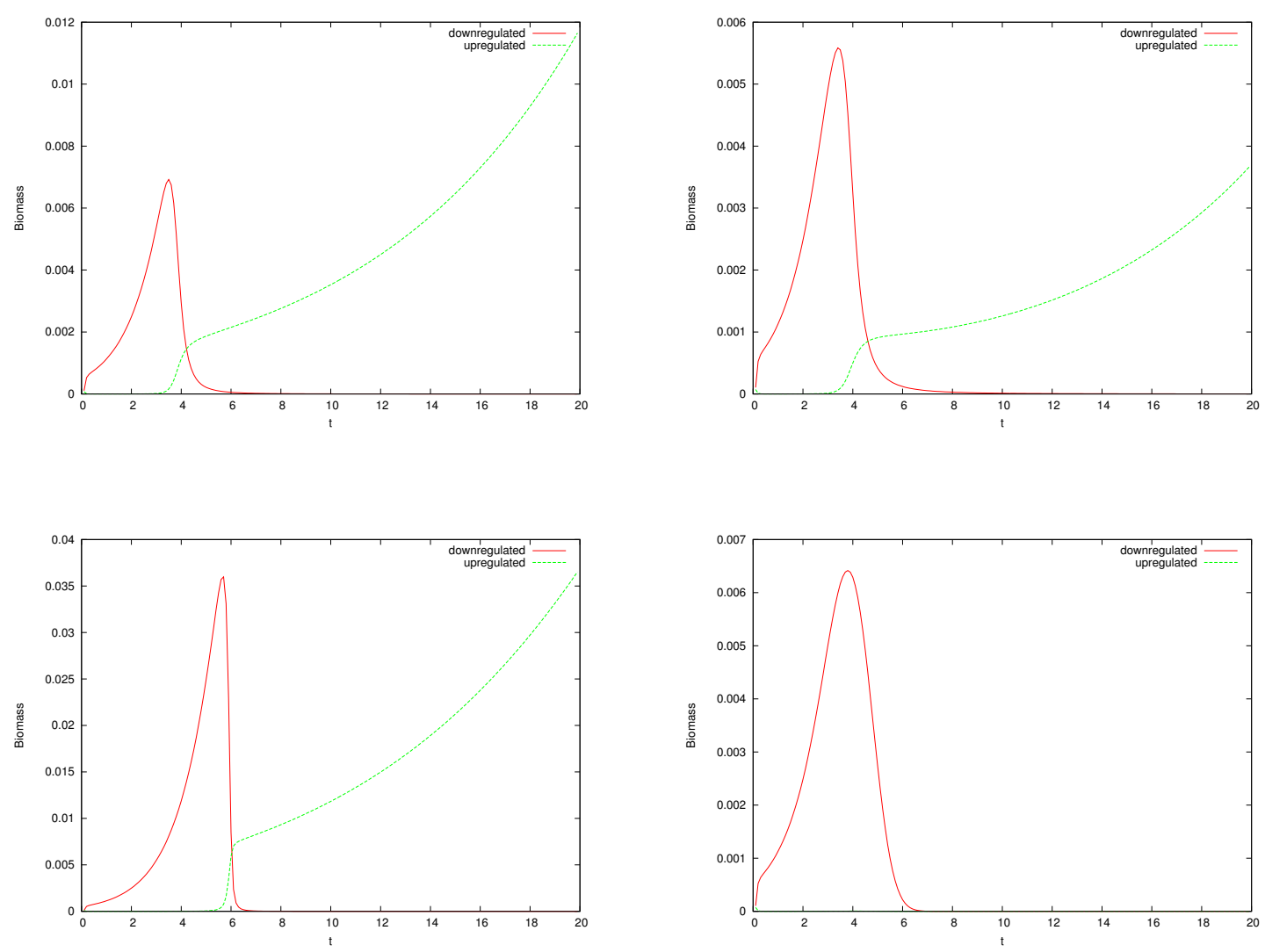

Figure 3. Total amounts of $X$ and $Y$ with respect to time. Top left: without antibiotic and QSB, Top right: with antibiotic, bottom left: with QSB and bottom right: with antibiotic and QSB.

In Figure 3 we plot the evolution of the total amount of the down-regulated and up-regulated biomass in different situations: without antibiotic and QSB, in presence of antibiotic, in presence of QSB and in presence of antibiotic and QSB. From the system without antibotic and QSB we can deduce that the switch from downregulated to up-regulated biomass occurs at $t \approx 4$. At this time, the down-regulated biomass starts to decrease whereas the up-regulated cells are produced. The production of up-regulated cells at this time is in agreement with the amount of AHL which increases more rapidly from $t=4$, see Figure 4 (right).

The presence of antibiotic reduces the total amount of biomass but the switch form down-regulated to upregulated cells occurs at $t \approx 4$ as well. The presence of QSB reduces the total amount of biomass and delays the switch form down-regulated to up-regulated cells that occurs at $t \approx 6$. This implies a bigger amount of down-regulated cells and then a bigger amount of up-regulated cells. In presence of antibiotic and QSB, the total amount of down-regulated cells is close to 0 for $t \geq 6$ and the total amount of up-regulated cells is about $10^{-5}$ for $4 \leq t \leq 6$ and is close to 0 for $t<4$ and $t>6$.

Figure 4 shows the evolution of the AHL molecule and the LasR protein with respect to time in the four cases: without antibiotic and QSB, with antibiotic, with QSB, with antibiotic and QSB. The increase of AHL is more important from $t \approx 4$ in the situation in which there is no antibiotic nor QSB, which is in accordance 
to the switch of down-regulated cells to up-regulated cells at this time. The total amount of AHL is lower in the presence of antibiotic whereas it is greater in the presence of QSB. This is due to the fact that the QSB increase the total biomass. When antibiotic and QSB act simultaneously $A_{\text {Total }}$ decreases significantly. The total amount of LasR protein is not modified by the antibiotic, but it reduces to $10^{-3}$ when the QSB is applied.
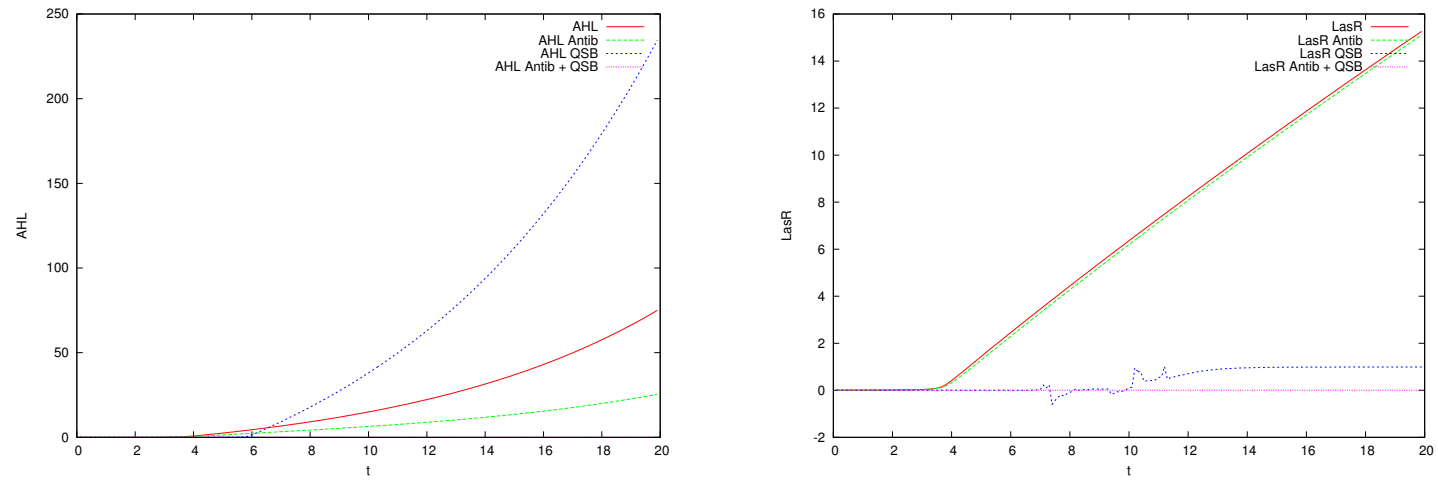

Figure 4. Comparison of the total amounts $A_{\text {Total }}$ and $R_{\text {Total }}$ in respect of time in the different four cases.

Figure 5 shows a comparison between different combination of dosing of the antibiotic and the QSB agent. The plot in Figures 3 and 4 underline the interest of using simultaneously an antibiotic and a QSB agent. In Figure 5 we plot $X_{\text {Total }}(t), Y_{\text {Total }}(t), A_{\text {Total }}(t)$ and $R_{\text {Total }}(t)$ when $V_{D}=Q_{D}=10, V_{D}=10, Q_{D}=5$, and $V_{D}=5, Q_{D}=10$.

One can observe that the total amount of up-regulated cells is more reduced when $Q_{D}>V_{D}$ than the case $V_{D}>Q_{D}$ despite the fact that down-regulated cells are less produced in the presence of a higher dose of antibiotic. This could be explained by the plot of the total amount of AHL which shows that AHL is significantly less produced when $V_{D}<Q_{D}$. The case $V_{D}<Q_{D}$ leads to a higher production of LasR protein than the cases where $V_{D}=10$. These results show that balancing between antibiotic and QSB dosing is not obvious. The study of different combination of the dosing will be the object of a forthcoming paper. 

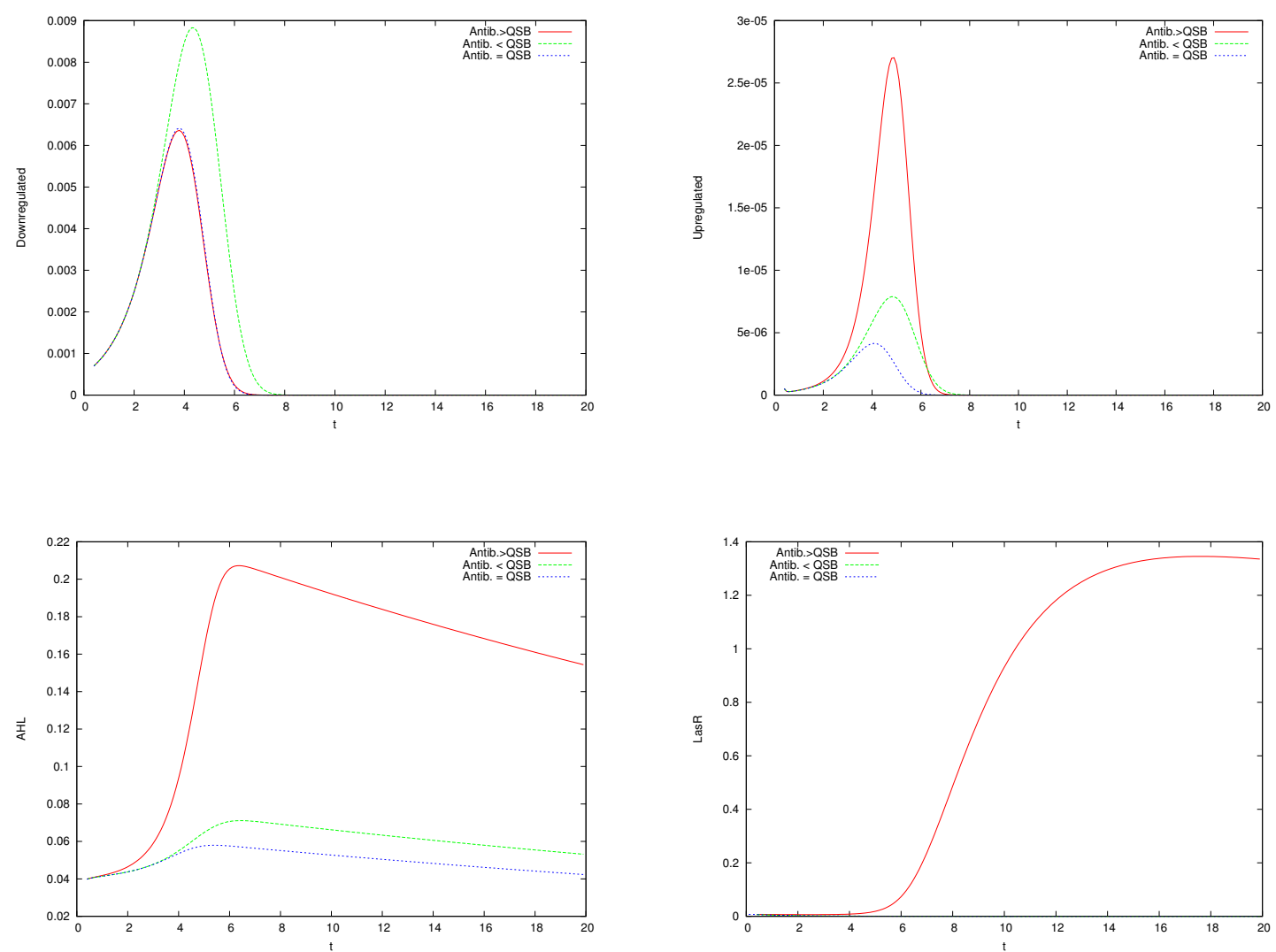

Figure 5. Comparison of the total amounts $A_{\text {Total }}$ and $R_{\text {Total }}$ with respect to time when $V_{D}=10, Q_{D}=5$ and $V_{D}=5, Q_{D}=10$. Top left: down-regulated cells, Top right: up-regulated cells, Bottom left: AHL and Bottom right: LasR.

\section{Conclusion}

We have considered a reaction-diffusion model describing the behavior of Pseudomenas aeruginosa bacteria and its signal molecules AHL. We have investigated the effects of anti-lasR and antibiotics on this system. Our qualitative study shows that an antibiotic treatment, alone, reduces the concentration of bacteria. And the LasR-blocking can delay the beginning virulence and thus leads to the increase of down-regulated bacteria and next the up-regulated one.

We also show that a combined treatment using an anti-LasR together with an antibiotic always reduce the LasR as well as the AHL concentrations to low levels. However, the qualitative response to treatment is very sensitive to parameter values. In forthcoming work we will perform a steady-state analysis of the governing equations to clarify the evolution of species and in particularly to how can we ensure non-trivial small stable steady state values for $R$ and $A$ for a given LasR-degradation rate. 


\section{ACKNOWLEDGEMENT}

The authors would like to thank the anonymous referee for suggestions resulting in significant improvement of the paper.

\section{REFERENCES}

[1] Anguige K, King J. R., Ward J. P., Williams P., Mathematical modelling of therapies targeted at bacterial quorum sensing, Math Biosci., 192(1), pp. 39-83, (2004).

[2] Anguige K., King J. R., Ward J. P., Modelling antibiotic- and anti-quorum sensing treatment of a spatially-structured Pseudomonas aeruginosa population, J Math Biol., 51(5):557-94, (2005).

[3] Barrett J. W., Nurnberg, R. Finite element approximation of a nonlinear degenerate parabolic system describing bacterial pattern formation, Interfaces and Free boundaries, 4, 277-300, (2002).

[4] Chopp, D. L., Kirisits, M. J., Parsek, M. R., And Moran B., A mathematical model of quorum sensing in a growing P. aeruginosa biofilm, Journal of Industrial Microbiology and Biotechnology. 29(6):339-346, (2002).

[5] Dockery, J. D., And Keener, J.P. A mathematical model for quorum sens- ing in P. Aeruginosa, Bulletin of Mathematical Biology. 63(1): 95-116, (2001).

[6] Frederick M. R., Kuttler C., Hense B. A., Eberl H. J., A mathematical model of quorum sensing regulated EPS production in biofilm communities, Theor Biol Med Model. (2011) Apr 10;8:8. doi: 10.1186/1742-4682-8-8.

[7] Sonnera S., Efendiev M. A., Eberl H. J. On the well-posedness of a mathematical model of quorum-sensing in patchy biofilm communities, (2011) (wileyonlinelibrary.com) DOI: 10.1002/mma.1475

[8] Sonnera S., Efendiev M. A., Eberl H. J. Existence and longtime behavior of a biofilm model, Communications on Pure and Applied Analysis. 8(2):509- 531, (2009).

[9] Wanner O., Gujer W. A multispecies biofilm model, Biotechnol Bioeng. 1986 Mar;28(3):314-28.

[10] Williams, P., Quorum sensing: an emerging target for antibacterial chemotherapy?, Expert Opin. Ther. Targets 6 257, (2002).

[11] Wood, B. D., And Whitaker, S. Cellular growth in biofilms, Biotechnology and Bioengineering, 64(6):656-670, (1999). 